\title{
Expression of the mceA, esat- 6 and $h s p X$ genes in Mycobacterium tuberculosis and their responses to aerobic conditions and to restricted oxygen supply
}

\author{
Yoseph Haile, ${ }^{1-3}$ Gunnar Bjune ${ }^{2,4}$ and Harald G. Wiker ${ }^{1}$
}

\begin{abstract}
Author for correspondence: Harald G. Wiker. Tel: +47220426 74. Fax: +4722042686. e-mail: harald.g.wiker@fhi.no
\end{abstract}

1 Norwegian Institute of Public Health, PO Box 4404, Nydalen, N-0403 Oslo, Norway

2 Center for International Health, University of Bergen, Armauer Hansens Hus, N-5021 Bergen, Norway

3 Broegelmann Research Laboratory, University of Bergen, Armauer Hansens Hus, N-5021 Bergen, Norway

${ }^{4}$ Institute of General Practice and Community Medicine, University of Oslo, PO Box 1130 Blindern, N-0317 Oslo, Norway

\begin{abstract}
The expression of six of the mammalian cell-entry (mce1a-mce1f) genes of the mce1 operon of Mycobacterium tuberculosis has been described previously. In this study, data are presented for the expression of other mammalian cellentry homologues (mce-2a, mce-3a and mce-4a) at the RNA level, as determined by RT-PCR. The stress responses of these genes and of other immunologically important antigens are also characterized with respect to the introduction of oxygen depletion. Analysis of the expression of the $m c e A$ genes in relation to oxygen depletion revealed that they were expressed differentially. The RT-PCR results showed that mce-1a, mce-2a, hspX (encoding the $\alpha$-crystallin antigen Acr) and esat- 6 (encoding the early secretory antigenic target-6) were expressed throughout the cultivation period, whereas the expression of mce3a and mce-4a was downregulated in the later stages of cultivation. This study gives new insights into the expression profiles of the different mce operons and the $h s p X$ and esat- 6 genes in an in vitro model of dormant-like bacilli. Identification of the genes that are differentially expressed under aerobic conditions and under oxygen-limited conditions contributes to our understanding of the bacilli involved in latent tuberculosis.
\end{abstract}

Keywords: mammalian cell entry, alpha-crystallin, RT-PCR

\section{INTRODUCTION}

Mycobacterium tuberculosis is able to escape the oxygen-dependent and oxygen-independent killing mechanisms of macrophages and to survive in host tissue without causing active disease for long periods of time (McKinney et al., 1998). Dormant bacilli remain quiescent in the host tissue without any sign of infection, and most cases of tuberculosis are due to the reactivation of dormant M. tuberculosis bacilli rather than to recent infection (Smith \& Moss, 1994). At present, one-third of the world's population is estimated to be latently infected with this pathogen, and about $10 \%$ of this population will develop reactivated tuberculosis during their lifetime. Currently, M. tuberculosis is responsible for 20 million active cases of tuberculosis and for three million deaths annually (Kochi, 1991). In general, the variability in the efficacy of the Bacille Calmette-Guérin (BCG) vaccination and its lack of significant effects against reactivated tuberculosis necessitate new strategies for the prevention of reactivation by elimination of dormant bacilli during latent infection. Identification of the genes that are expressed during dormancy is a fundamental goal if new vaccines and anti-mycobacterial drugs targeted against the pathogen when it is in this stage of infection are to be considered.

The survival mechanism of dormant tubercle bacilli is still obscure. Different studies have been conducted to elucidate the nature of this dormant state. Clinical and experimental evidence suggest that mycobacterial persistence might arise in response to different stress responses, such as oxygen limitation (Wayne \& Lin, 1982). In addition, oxygen-starved tubercle bacilli are rapidly killed by the drug metronidazole, which is usually used for the treatment of infections caused by anaerobic microbes (Wayne \& Sramek, 1994). Attempts have been made to identify and characterize genes expressed under stress conditions. Variations in antigen composition in vitro between non-dividing and actively growing bacilli have been demonstrated (Wayne \& Sramek, 1979). Protein analysis of cultures grown under stress conditions has demonstrated the accumulation of an $\alpha$-crystallin-like protein (Acr) in mid-exponential and 
Y. Haile, G. Bjune and H. G. Wiker

Table 1. Primer pairs used for CDNA synthesis and RT-PCR

\begin{tabular}{|c|c|c|c|}
\hline Pair no. & Gene & $\begin{array}{l}\text { Direction/position } \\
\text { of primer }\end{array}$ & Sequence \\
\hline \multirow[t]{2}{*}{1} & $m c e-1 a$ & Forward, 225-245 & 5'-CAC CAT CTC GGA GGT CAC ACG-3' \\
\hline & & Reverse, 520-501 & 5'-CGC TCA GGG TCA GGT TCA GC-3' \\
\hline \multirow[t]{2}{*}{2} & $m c e-2 a$ & Forward, 117-136 & 5'-ATT CAC GCC GAA GAC CGA GC-3' \\
\hline & & Reverse, 430-411 & 5'-CCG ACC CCA CAT CAA TCA CG-3' \\
\hline \multirow[t]{2}{*}{3} & $m c e-3 a$ & Forward, 529-548 & 5'-GGG CAA GGT GAA GGC ATA GG-3' \\
\hline & & Reverse, 883-864 & 5'-GGA AGC AGG TGT ATT CGG GG-3' \\
\hline \multirow[t]{2}{*}{4} & $m c e-4 a$ & Forward, 190-210 & 5'-GGC ATC CAG GTA GGC AAG GTC-3' \\
\hline & & Reverse, 569-550 & 5'-CGG GTC AGC GTG TTC AGT CC-3' \\
\hline \multirow[t]{2}{*}{5} & esat-6 & Forward, 71-91 & 5'-CCA TTC ATT CCC TCC TTG ACG-3' \\
\hline & & Reverse, 285-267 & 5'-TGC GAA CAT CCC AGT GAC G-3' \\
\hline \multirow[t]{2}{*}{6} & $h s p X$ & Forward, 126-146 & 5'-GGA AGA CGA GAT GAA AGA GGG G-3' \\
\hline & & Reverse, 306-286 & 5'-AAC CGC CAC CGA CAC AGT AAG-3' \\
\hline \multirow[t]{2}{*}{7} & $16 \mathrm{~S}$ rRNA & Forward, 469-491 & 5'-TTG ACG GTA GGT GGA GAA GAA GC-3' \\
\hline & & Reverse, 909-888 & 5'-CCT TTG AGT TTT AGC CTT GCG G-3' \\
\hline
\end{tabular}

stationary phase cultures of $M$. tuberculosis (Yuan et al., 1996). Experimental evidence has also indicated that Acr is needed for the growth of M. tuberculosis in cultured macrophages (Yuan et al., 1998). However, the genetic factor(s) responsible for dormancy are still not known. One of the assumptions is that adaptation of the bacilli to long-term survival under stress conditions may be associated with coordinated alterations in the pattern of gene expression. Alternatively, the entry and invasion mechanisms of the bacilli might be associated with their survival under unfavourable conditions. In addition, recent findings indicate that $M$. tuberculosis escapes from unfavourable conditions by hiding in non-professional phagocytic cells as well as by evading the macrophage response (Hernandez-Pando et al., 2000). Arruda et al. (1993) have shown that a cloned mammalian cell-entry gene $(m c e-1 a)$ from $M$. tuberculosis conferred upon non-pathogenic Escherichia coli the ability to invade and survive inside a human cancer cell line (HeLa) and inside macrophages. This gene is associated with two integral proteins and is succeeded by five genes, and is assumed to be important for the entry and survival of M. tuberculosis in the host cell. Information gained from the $M$. tuberculosis wholegenome sequence has revealed the presence of four homologous mce operons, termed mce1, mce2, mce3 and mce4 (Cole et al., 1998). One of the possible explanations for the duplication of mce is the variation in the regulation of each operon (Ahmad et al., 1999). Investigating the expression profiles of the mce genes in each of the operons in relation to oxygen depletion might give an overview as to how the transcription of these homologous operons is coordinated. As described by Haile et al. (2002), alignment of the deduced Mce1A, Mce2A, Mce3A and Mce4A proteins showed that Mce1A has $65.7 \%$ similarity with Mce2A, and that the Mce3A and Mce4A proteins are comparatively diverged from Mce1A and Mce2A, with a maximum of $32.5 \%$ sequence similarity.
Although the expression of all six of the mce1 genes of the $m c e 1$ operon has been demonstrated by Harboe et al. (1999), the regulatory mechanisms of these genes are largely unknown. In this study, we have looked at the relationship between in vitro actively growing and nondividing tubercle bacilli with regard to the expression profiles produced by the different mce operons, using the third gene of each operon as the representative for the operon. In addition, we have studied $h s p X$ (encoding the M. tuberculosis $16 \mathrm{kDa} \alpha$-crystallin antigen Acr) and esat-6 (encoding the early secretory antigenic target-6). Acr is known to accumulate in stationary and midexponential phase cultures (Yuan et al., 1996) of $M$. tuberculosis; ESAT-6 is an important antigen that is primarily expressed during the early phase of growth (Andersen et al., 1991) and is recognized by human Band T-cells (Harboe et al., 1998; Ulrichs et al., 1998; Mustafa et al., 2000).

\section{METHODS}

Preparation of stock culture. A colony of M. tuberculosis H37Rv taken from a Loewenstein-Jensen medium slant was inoculated into $10 \mathrm{ml}$ of Dubos broth containing albumindextrose complex (ADC) (Difco) and cultured for 10 days at $37^{\circ} \mathrm{C}$ with shaking at $12 \mathrm{~h}$ intervals. The culture was then inoculated into $200 \mathrm{ml}$ of Dubos/ADC medium and incubated as a stationary culture, which was shaken once a day. The culture was kept at $37^{\circ} \mathrm{C}$ for $10-14$ days, until it reached midexponential phase (about $10^{7}$ bacilli $\mathrm{ml}^{-1}$ ) and appeared cloudy. The number of bacilli present in the culture was estimated by using the McFardland standard and counts in a Toma chamber after acid-fast staining. The bacilli were then distributed into $50 \mathrm{ml}$ falcon tubes and kept as stock cultures at $-70{ }^{\circ} \mathrm{C}$ until required.

Preparation of cultures for restricted oxygen supply. Frozen cultures were subcultured into fresh medium $(1: 10, \mathrm{v} / \mathrm{v})$. Experiments were done by transferring cultures with actively growing bacilli into $50 \mathrm{ml}$ falcon tubes in a $2: 1$ air-to-medium (space) ratio; the cultures were incubated without shaking at 
$37^{\circ} \mathrm{C}$ for up to 2 months. Assessment of cell viability was done at $7,10,20,30,40$ and 60 days culture, by determining the c.f.u. counts on solid medium as described previously (Phyu et al., 1998).

Recovery of mycobacteria and isolation of RNA. Bacterial pellets were recovered at different time points from $20 \mathrm{ml}$ samples of the culture medium by centrifugation at $5000 \mathrm{~g}$ for $10 \mathrm{~min}$. RNA was then isolated from the cell pellets by using the combination of a shaking device (Hybaid) and Trizol RNA isolation buffer (Life Technologies) in a Hybaid ribolyser blue matrix (Hybaid), consisting of acid-washed, RNase-free silica/ceramic beads (Hybaid). Trizol RNA isolation buffer $(1 \mathrm{ml})$ and $100 \mu \mathrm{l}$ cell pellet were added to the matrix prior to the disruption of the cell wall by sonication for $45 \mathrm{~s}$ (speed 6.5). The resulting lysate was mixed with $200 \mu \mathrm{l}$ chloroform, shaken vigorously for $10 \mathrm{~s}$ and kept at room temperature for $2 \mathrm{~min}$. It was then centrifuged at $12000 \mathrm{~g}$ for $30 \mathrm{~min}$ at $4{ }^{\circ} \mathrm{C}$, to separate the cell debris from the supernatant. The upper aqueous phase, which contained the total RNA, was carefully pipetted into an Eppendorf tube. Subsequently, $500 \mu \mathrm{l} \mathrm{2-}$ propanol was added to the aqueous phase, and the mixture was incubated at room temperature for $10 \mathrm{~min}$. The RNA was then pelleted by centrifugation at $12500 \mathrm{~g}$ for $15 \mathrm{~min}$ at $4{ }^{\circ} \mathrm{C}$. The supernatant was discarded and the RNA pellet was washed once with $75 \%(\mathrm{v} / \mathrm{v})$ ethanol. The ethanol was removed from the pellet after centrifugation at $7500 \mathrm{~g}$ for $5 \mathrm{~min}$; the pellet was then air-dried and redissolved in $50 \mu \mathrm{l}$ RNase-free water and the RNA concentration was determined spectrophotometrically at $260 \mathrm{~nm}$.

Primers for CDNA synthesis and RT-PCR. A pair of primers for each gene was designed and ordered from MedProbe. The sequences for the primers used in this study are listed in Table 1 and are given in the $5^{\prime} \rightarrow 3^{\prime}$ direction.

Removal of genomic DNA and cDNA synthesis. To remove genomic DNA, the RNA was treated with RNase-free DNaseI (Life Technologies) prior to cDNA synthesis. Five millilitres of $10 \times$ DNaseI buffer and 5 U RNase-free DNaseI were added to $50 \mu \mathrm{l}$ total RNA and then incubated at room temperature for $15 \mathrm{~min}$. The nuclease activity was stopped by adding $1 \mu \mathrm{l}$ of $25 \mathrm{mM}$ EDTA ( $\mathrm{pH} 8$ ) to the reaction mixture, which was then incubated at $65^{\circ} \mathrm{C}$ for $10 \mathrm{~min}$.

cDNA was then synthesized from $0.5 \mu \mathrm{g}$ of DNaseI-treated total RNA in a total reaction volume of $30 \mu \mathrm{l}$, using avian myeloblastosis virus (MLV) reverse transcriptase (Promega). The reaction mixture contained reverse transcriptase buffer, a dNTP mix $(0.25 \mathrm{mM}$ each of dATP, dCTP, dTTP and dGTP), $1 \mathrm{mM}$ reverse primer, $0.6 \mathrm{U}$ RNase inhibitor (Promega) and $25 \mathrm{U}$ MLV reverse transcriptase. The mixture was incubated at $42{ }^{\circ} \mathrm{C}$ for $50 \mathrm{~min}$ in a waterbath, heated to $95^{\circ} \mathrm{C}$ for $5 \mathrm{~min}$ to inactivate the enzyme and then chilled on ice. Finally, $70 \mu \mathrm{l}$ RNase-free water was added to the mixture and the sample was stored at $-20^{\circ} \mathrm{C}$ until used for PCR.

PCR. To choose the right concentration of cDNA for semiquantitative analysis, a 10 -fold serial dilution of the cDNA was made. PCR was then performed in a total volume of $25 \mu \mathrm{l}$, using an automated GeneAMP system 9700 thermal cycler (Perkin Elmer). The reaction mixture contained PCR buffer (10 mM Tris/HCl, pH 8.3, $3 \mathrm{mM} \mathrm{MgCl}_{2}, 50 \mathrm{mM} \mathrm{KCl}$ ), a dNTP mix $(0 \cdot 2 \mathrm{mM}$ each of dATP, dCTP, dGTP and dTTP), $1 \mu \mathrm{M}$ of the reverse and the forward primer and 1.6 U Taq DNA polymerase (Promega). The amplification procedure involved initial denaturation at $94^{\circ} \mathrm{C}$ for 4 min followed by 35 cycles of denaturation at $94^{\circ} \mathrm{C}$ for $1 \mathrm{~min}$, annealing of primers at $60{ }^{\circ} \mathrm{C}$ for $1 \mathrm{~min}$ and primer extension at $72{ }^{\circ} \mathrm{C}$ for $30 \mathrm{~s}$. After completion of the 35 th cycle, the extension reaction was continued for another $10 \mathrm{~min}$ at $72{ }^{\circ} \mathrm{C}$. The positive control consisted of $M$. tuberculosis genomic DNA; the negative control consisted of the reaction mixture minus the cDNA. We chose $16 \mathrm{~S}$ rRNA as the internal control, based on the assumption that the presence of intact $16 \mathrm{~S}$ rRNA is associated with the presence of live bacilli; $16 \mathrm{~S}$ rRNA is also highly abundant in cells.

\section{RESULTS}

\section{Establishment of oxygen restriction in the culture}

Based on c.f.u. counts, the bacilli replicated from day 0 up to day 20. The number of proliferating bacilli remained at the same level between days 40 and 60, after which time the number started to decrease. The cultures appeared different at the different stages of growth. As observed by $\mathrm{Hu} \&$ Coates (1999), bacilli were found exclusively at the surface of the culture medium until about day 10 , whereupon they started to sink down in the medium. Within 2 months of inoculation, all of the bacilli appeared as sediment at the bottom of the culture vessel. In our culture system, the bacilli remained on the surface of the medium until day 20 and then started to sink down the medium, indicating that bacilli had entered into a non-replicating stage. Since the cultures were sealed and not agitated, the amount of available oxygen decreased; hence, the cultures gradually transformed from being oxygen-rich to hypoxic. We chose cultures incubated for 7-10 days, 20-40 days and 2 months to investigate the expression of the mce-1a, $m c e-2 a$, mce-3a, mce-4a, hspX and esat-6 genes in actively replicating and non-replicating cultures of $M$. tuberculosis.

\section{Assessment of transcription of the different mce genes}

To assess the transcription of the mce-1a, mce- $2 a$, mce$3 a$, mce- $4 a$, hspX and esat-6 genes, total RNA was isolated from an actively replicating culture of $M$. tuberculosis and subjected to DNaseI treatment. Reverse primers were used for CDNA synthesis, which was then amplified by PCR using specific primers for each gene. The transcription of mRNA from esat-6, hspX and the mce operons as determined by RT-PCR is shown in Fig. 1. The negative control showed that the bands produced in the test samples were from M. tuberculosis.

\section{Standardization of the total RNA and differential expression of the different $m c e A$ genes}

To assess the presence of any inhibitory substances and for the normalization of the RNA in different samples we chose 16S rRNA as the internal control. The presence of non-degraded $16 \mathrm{~S}$ rRNA under actively replicating and non-replicating conditions was used as the criterion for the presence of live bacilli (10000-100000 copies of $16 \mathrm{~S}$ rRNA per cell). cDNA was synthesized from $0.5 \mu \mathrm{g}$ of total RNA isolated from M. tuberculosis at 7, 10, 20, 30, 40 and 60 days of culture by using the 16S rRNA reverse primer (Table 1). PCR was performed on DNA 


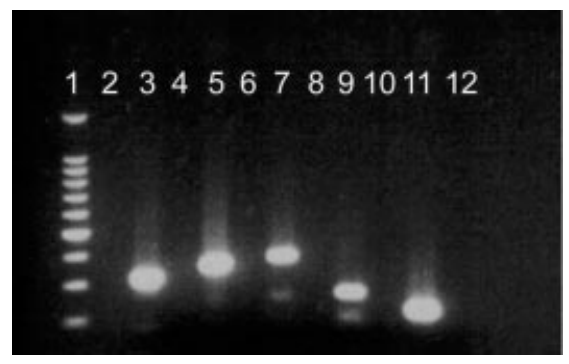

Fig. 1. Expression of the mce-2a, mce-3a, mce-4a, esat- 6 and hspX genes, as determined by RT-PCR. Lanes: 1 , molecular mass marker; 2, empty well; 3, mce-2a RNA; 4, mce-2a negative control; 5, mce-3a RNA; 6, mce-3a negative control; 7, mce-4a RNA; 8 , mce-4a negative control; 9 , esat-6 RNA; 10 , esat-6 negative control; 11 , $h s p X$ RNA; 12 , hspX negative control.

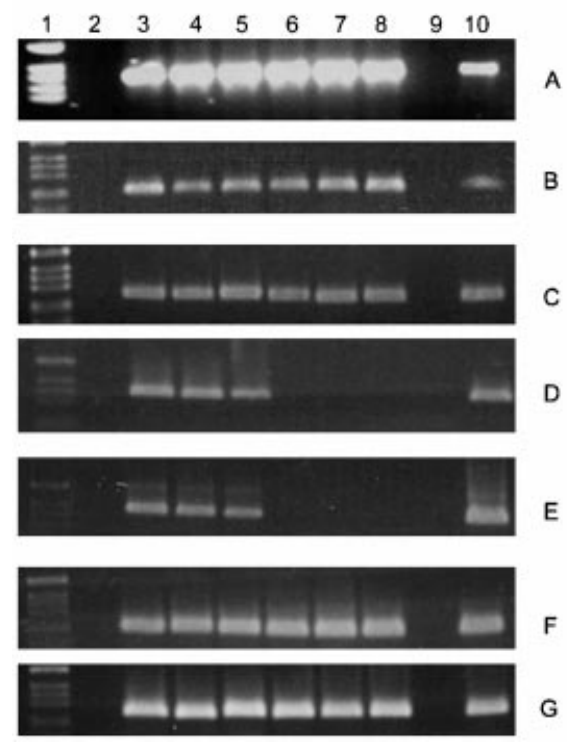

Fig. 2. RT-PCR products obtained using the seven primer pairs detailed in Table 1. (A) M. tuberculosis $16 \mathrm{~S}$ rRNA, (B) mce-1a, (C) mce-2a, (D) mce-3a, (E) mce-4a, (F) esat-6 and (G) hspX. The cultures were grown in Dubos broth for $7,10,20,30,40$ and 60 days. Lanes: 1, molecular mass marker; 2, empty well; 3, RNA extracted after 7 days culture; 4, RNA extracted after 10 days culture; 5, RNA extracted after 20 days culture; 6, RNA extracted after 30 days culture; 7, RNA extracted after 40 days culture; 8 , RNA extracted after 60 days culture; 9, negative control; 10, positive control. The same $M$. tuberculosis culture was used for analysis of all of the genes at each time point.

extracted from the same cultures by using the $16 \mathrm{~S}$ rRNA primer pair (Table 1). Similar levels of $16 \mathrm{~S}$ rRNA were observed throughout the whole culture period (Fig. 2); this result ruled out the presence of strong inhibitory substances in the culture medium.

To assess the expression of esat-6, hspX and the mceA genes with increasing oxygen depletion, we extracted mRNA at different time points (i.e. at 7, 10, 20, 30, 40 and 60 days of culture) and performed RT-PCR. Up until the 20th day of culture all of the genes investigated here were expressed. mce-1a, mce-2a and $h s p X$ were expressed in the actively growing and non-replicating cultures (Fig. 2). We also observed expression of $h s p X$ in the actively growing culture at 7 and 10 days of culture. esat-6 was expressed in the actively growing and nonreplicating cultures (Fig. 2). We performed RT-PCR for $16 \mathrm{~S}$ rRNA on the same RNA sources as used above and obtained a positive signal at all time points (Fig. 2). Visual inspection of the stained gels showed that the RT-PCR products for $m c e-1 a$ and $m c e-2 a$ in the actively growing and non-replicating cultures of $M$. tuberculosis were present at similar levels.

One of the striking findings from this study was that expression of both $m c e-3 a$ and $m c e-4 a$ was positive on the 7 th, 10th and 20th day of culture. There was a very weak positive signal for the expression of $m c e-4 a$ at the 40th day of culture, but this was only revealed by densitometry. Otherwise, there was no expression of $m c e-3 a$ or $m c e-4 a$ in the oxygen-depleted phase, although $16 \mathrm{~S}$ rRNA was present during this phase (Fig. 2).

\section{DISCUSSION}

Assessment of the stress responses of M. tuberculosis at the molecular level is still in its infancy. Antigenic differences between extracts from actively growing and non-replicating $M$. tuberculosis cells have been described previously (Wayne \& Sramek, 1979). In this study, we defined actively replicating and non-replicating cultures of $M$. tuberculosis on the basis of culture characteristics and c.f.u. counts of the bacilli. A large inoculum might lead to an early shiftdown of oxygen availability and to the induction of genes that are expressed under stress conditions. However, in our experiments we used a small initial inoculum. The 10fold increase in the c.f.u. count of the bacilli indicated the presence of enough oxygen and nutrients in the medium for multiplication of the bacilli in the initial stages of culture. Research efforts into understanding the effects of oxygen deprivation on in vitro dormantlike bacilli have shown a direct relationship between dissolved oxygen, DNA synthesis and the concentration of glycogen dehydrogenase present (Wayne \& Hayes, 1996). In our experiments, the stable c.f.u. counts observed after day 20 of cultivation could be an effect of oxygen depletion in the unagitated culture in addition to the nutrient deprivation and decreased $\mathrm{pH}$. Wayne \& Lin (1982) showed that vegetatively growing M. tuberculosis cells switch their metabolism to the glyoxylate pathway to survive under anaerobic conditions and form a sediment at the bottom of the culture vessel, whereas actively growing bacilli stay at the surface of the culture medium where they get enough oxygen for multiplication because oxygen access is not limited. In our experiments, all of the bacilli remained at the surface of the culture medium in the replicating stage of growth and no bacilli were present at the bottom of the culture vessel. After 20 days culture, the bacilli started to sink down the medium; after 40 days culture all of the bacteria were found at the bottom of the culture vessel, indicating that oxygen was about to be depleted. 
Contamination by genomic DNA in the total RNA preparation leads to false positive results. In our study, the presence of genomic DNA was less likely to influence results because we used DNaseI-treated total RNA rather than immediate cDNA synthesized from RNA in the RT-PCR procedure. We were also able to amplify $16 \mathrm{~S}$ rRNA, the control, from all of our test samples but not from the negative control, indicating that inhibitors were not causing false negative results in our experiments.

Previous experiments have indicated that mce-1a is required for entry into and survival of M. tuberculosis in the host cell (Aruda et al., 1993). An expression study has indicated that polyvalent rabbit antibodies generated by immunization with sonicates of BCG bacilli reacted with synthetic peptides from the six proteins (Mce1a-Mce1f) translated from the mce1 operon (Harboe et al., 1999). Now, using the third gene as the representative for each operon, we have shown expression of the M. tuberculosis mce-2a, mce-3a and mce$4 a$ genes. In addition to demonstrating their expression, we also noticed differential regulation of the different $m c e A$ genes in response to oxygen depletion. Downregulation of the mce-3a and mce- $4 a$ genes may start earlier in the growth phase, but it was not noticed here due to the sensitivity of the RT-PCR technique. Whether this is the case remains to be elucidated, but our results do indicate that almost complete downregulation of expression of $m c e-3 a$ and $m c e-4 a$ was achieved by 30 days culture.

Several explanations and suggestions could be forwarded from these observations. Besides the wide distribution of the four mce operons in mycobacteria other than tuberculosis (MOTT) (Haile et al., 2002; Wiker et al., 1999), the expression of all the mceA genes in actively growing M. tuberculosis cultures indicates their functional importance in the entry and survival of the bacilli in the host cell. Further expressional studies at the protein level of the mce operons in MOTT will help to clarify this point.

Alternatively, the pathogenicity of M. tuberculosis might depend on all of the genes in the different mce operons working together or different stimuli could activate different genes in the same or different operons. In this regard, the invariable expression of $m c e-1 a$ and mce-2a might reflect the importance of both of these genes in actively growing and non-replicating dormantlike bacilli, while mce-3a and mce-4a might be functionally important only in actively growing bacilli. Realtime quantitative PCR may be used to identify more exactly the time point and conditions that initiate downregulation of mce-3a and mce-4a. While oxygen depletion was used to study gene expression in nonreplicating bacilli in vitro in this work, further studies are necessary to determine whether downregulation of $m c e-3 a$ and $m c e-4 a$ is associated with dormancy in vivo. Expression of the mceA genes is not necessarily representative of the expression of the other genes within each of the respective operons. We have previously shown that the six genes of the mce1 operon, mcela- mce1f, are expressed upon in vitro culture of $M$. tuberculosis (Harboe et al., 1999), but further expression studies on genes $m c e B-m c e F$ are needed to clarify whether these genes are upregulated or downregulated upon oxygen depletion.

One of the controversies with regard to the dormancy of M. tuberculosis is whether dormant M. tuberculosis bacilli are present only in oxygen-depleted granulomas. Different experiments have suggested that hypoxic conditions occur concomitantly with granuloma formation and that the bacilli downregulate the expression of many genes under hypoxic conditions and enter into non-replicating, dormant stages. Recent data have suggested that dormant bacilli are found not only in macrophages of granulomas but also in non-professional phagocytic cells. Dormant bacilli have been isolated from normal-looking lung biopsies (HernandezPando et al., 2000). This controversy can only be resolved by further analyses of tissue samples.

Finally, the levels of expression of the different mce genes might be important for evaluating their putative functions. Quantitative analysis will give information about the relative expression of the different mce genes and will also provide information for genes that have a function in the regulation of transcription. The ORFs $R v 0165 c, R v 0586, R v 1963 c$ and $R v 3522$ have been suggested to have a transcriptional regulatory function and may be involved in regulation of the mce operons, as they are found upstream of the operons mce1, mce2, mce 3 and mce4, respectively (Cole et al., 1998).

$h s p X$ is known to be strongly induced by mildly hypoxic conditions (Yuan et al., 1996). Unlike the studies by Sherman et al. (2001), Desjardin et al. (2001) found hspX to be expressed in both aerobic and stationary phase cultures. As explained previously by Florczyk et al. (2001), the differences in bacterial culture conditions have important implications for the study of gene expression in mycobacteria. In addition, RNA analysis is more sensitive than protein analysis, and this might explain why we detected $h s p X$ in the early growth stages of the M. tuberculosis cultures used here. We also found esat-6 to be expressed in non-replicating bacilli, indicating that this gene is active not only in the early phase of culture (Sørensen et al., 1995) but also in the late phase. Quantitative transcriptional analyses of the $h s p X$ and esat-6 genes might be required to fully understand the expression of these genes in the early and late phases of culture.

Since oxygen deprivation was associated with a depletion in the levels of mce-3a and mce-4a expression, the presence of $m c e-3 a$ and $m c e-4 a$ mRNA in actively growing bacilli suggests a possibility for distinguishing between the active disease and latent tuberculosis.

\section{ACKNOWLEDGEMENTS}

We thank Professor Bjarne Bjorvatn, University of Bergen, for his kind permission to use the facilities of the Mycobacteriology Laboratory for the cultivation of M. tuberculosis. We would also like to acknowledge Fesseha Abebe and Sabai 
Phyu for their help in the cultivation and counting of mycobacteria. This work was supported by the Center for International Health and the Broegelmann Research Laboratory, University of Bergen, Norway.

\section{REFERENCES}

Ahmad, S., Akbar, P. K., Wiker, H. G., Harboe, M. \& Mustafa, A. S. (1999). Cloning, expression and immunological reactivity of two mammalian cell entry proteins encoded by the mce 1 operon of Mycobacterium tuberculosis. Scand J Immunol 50, 510-518.

Andersen, P., Askgaard, D., Ljungqvist, L., Bennedsen, J. \& Heron, I. (1991). Proteins released from Mycobacterium tuberculosis during growth. Infect Immun 59, 1905-1910.

Arruda, S., Bomfim, G., Knights, R., Huima-Byron, T. \& Riley, L. W. (1993). Cloning of an M. tuberculosis DNA fragment associated with entry and survival inside cells. Science 261, 1454-1457.

Cole, S. T., Brosch, R., Parkhill, J. \& 39 other authors. (1998). Deciphering the biology of Mycobacterium tuberculosis from the complete genome sequence. Nature 393, 537-544.

Desjardin, L. E., Hayes, L. G., Sohaskey, C. D., Wayne, L. G. \& Eisenach, K. D. (2001). Microaerophilic induction of the $\alpha$ crystallin chaperone protein homologue ( $h s p X)$ mRNA of $M y c o-$ bacterium tuberculosis. J Bacteriol 183, 5311-5316.

Florczyk, M. A., McCue, L. A., Stack, R. F., Hauer, C. R. \& McDonough, K. A. (2001). Identification and characterization of mycobacterial proteins differentially expressed under standing and shaking culture conditions, including Rv2623 from a novel class of putative ATP-binding proteins. Infect Immun $\mathbf{6 9}$, 5777-5785.

Haile, Y., Caugant, D. A., Bjune, G. \& Wiker, H. G. (2002). Mycobacterium tuberculosis mammalian cell entry operons (mce) homologs in Mycobacterium other than tuberculosis (MOTT). FEMS Immunol Med Microbiol 33, 125-132.

Harboe, M., Malin, A. S., Dockrell, H. S., Wiker, H. G., Ulvund, G., Holm, A., Jorgensen, M. C. \& Andersen, P. (1998). B-cell epitopes and quantification of the ESAT-6 protein of Mycobacterium tuberculosis. Infect Immun 66, 717-723.

Harboe, M., Christensen, A., Haile, Y., Ulvund, G., Ahmad, S., Mustafa, A. S. \& Wiker, H. G. (1999). Demonstration of expression of six proteins of the mammalian cell entry (mce1) operon of Mycobacterium tuberculosis by anti-peptide antibodies, enzymelinked immunosorbent assay and reverse transcription-polymerase chain reaction. Scand J Immunol 50, 519-527.

Hernandez-Pando, R., Jeyanathan, M., Mengistu, G., Aguilar, D., Orozco, H., Harboe, M., Rook, G. A. \& Bjune, G. (2000). Persistence of DNA from Mycobacterium tuberculosis in superficially normal lung tissue during latent infection. Lancet 356, 2133-2138.

Hu, Y. \& Coates, R. M. (1999). Transcription of two sigma 70 homologue genes, $\operatorname{sig} A$ and $\operatorname{sig} B$, in stationary-phase Mycobacterium tuberculosis. J Bacteriol 181, 469-476.

Kochi, A. (1991). The global tuberculosis situation and the new control strategy of the World Health Organization. Tubercle $\mathbf{7 2}$, $1-6$
McKinney, J. D., William, R., Jakobs, J. R. \& Bloom, B. R. (1998). Persisting problem in tuberculosis. In Tuberculosis, pp. 51-146. London: Acadamic press.

Mustafa, A. S., Oftung, F., Amoudy, H. A., Madi, N. M., Abal, A. T., Shaban, F., Rosen Krands, I. \& Andersen, P. (2000). Multiple epitopes from the Mycobacterium tuberculosis ESAT-6 antigen are recognized by antigen-specific human $\mathrm{T}$ cell lines. Clin Infect Dis 30 (suppl. 3), S201-S205.

Phyu, S., Mustafa, T., Hofstad, T., Nilsen, R., Fosse, R. \& Bjune, G. (1998). A mouse model for latent tuberculosis. Scand J Infect Dis 30, 59-68.

Sherman, D. R., Voskuil, M., Schnappinger, D., Liao, R., Harrell, M. I. \& Schoolinik, G. K. (2001). Regulation of the Mycobacterium tuberculosis hypoxic response gene encoding $\alpha$-crystallin. Proc Natl Acad Sci U S A 98, 7534-7539.

Smith, P. G. \& Moss, A. R. (1994). Epidemiology of tuberculosis. In Tuberculosis: Pathogenesis, Protection and Control. Edited by B. R. Bloom. Washington, DC: American Society for Microbiology.

Sørensen, A. L., Nagai, S., Houen, G., Andersen, P. \& Andersen, A. B. (1995). Purification and characterization of low-molecularmass T-cell antigen secreted by Mycobacterium tuberculosis. Infect Immun 63, 1710-1717.

Ulrichs, T., Munk, M. E., Mollenkopft, H., Behr-Perst, S., Colangeli, R., Gennaro, M. L. \& Kaufmann, S. H. (1998). Differential T cell responses to Mycobacterium tuberculosis ESAT-6 in tuberculosis patients and healthy donors. Eur J Immunol 28, 3949-3958.

Wayne, L. G. \& Hayes, L. G. (1996). An in vitro model for sequential study of shiftdown of Mycobacterium tuberculosis through two stages of nonreplicating persistence. Infect Immun 64, 2062-2069.

Wayne, L. G. \& Lin, K. Y. (1982). Glyoxylate metabolism and adaptation of Mycobacterium tuberculosis to survival under anaerobic conditions. Infect Immun 37, 1042-1049.

Wayne, L. G. \& Sramek, H. A. (1979). Antigenic differences between extracts of actively replicating and synchronized resting cells of Mycobacterium tuberculosis. Infect Immun 24, 363-370.

Wayne, L. G. \& Sramek, H. A. (1994). Metronidazole is bactericidal for the dormant cells of Mycobacterium tuberculosis. Antimicrob Agents Chemother 38, 2054-2058.

Wiker, H. G., Spierings, E., Kolkman, M. A., Ottenhoff, T. H. \& Harboe, M. (1999). The mammalian cell entry operon 1 (mce1) of Mycobacterium leprae and Mycobacterium tuberculosis. Microb Pathog 27, 173-177.

Yuan, Y., Crane, D. D. \& Barry, C. E., III (1996). Stationary phaseassociated protein expression in Mycobacterium tuberculosis: function of the mycobacterial $\alpha$-crystallin homolog. J Bacteriol 178, 4484-4492.

Yuan, Y., Crane, D. D., Simpson, R. M., Zhu, Y. Q., Hickey, M. J., Sherman, D. R. \& Barry, C. E., III (1998). The $16-k D a ~ \alpha-$ crystallin (Acr) protein of Mycobacterium tuberculosis is required for the growth in macrophages. Proc Natl Acad Sci U S A 95, 9578-9583.

Received 20 June 2002; revised 17 July 2002; accepted 27 August 2002. 